TITLE:

\title{
Visual Matrix Calculator For Undergraduate Students
}

$\operatorname{AUTHOR}(S)$ :

Uchida, Satoshi; Yamaki, Naokazu

CITATION:

Uchida, Satoshi ... [et al]. Visual Matrix Calculator For Undergraduate Students. 数理解析研 究所講究録 1998, 1040: 245-248

ISSUE DATE:

1998-04

URL:

http://hdl.handle.net/2433/62014

RIGHT: 


\title{
Visual Matrix Calculator For Undergraduate Students
}

\author{
Satoshi Uchida（内田智史) \\ Dept. of Industrial Engineering and Management \\ Kanagawa University \\ uchida@inf.ie.kanagawa-u.ac.jp \\ Naokazu Yamaki (八巻直一) \\ Dept. of Systems Engineering \\ Shizuoka University \\ yamaki@sys.eng.shizuoka.ac.jp
}

\section{Introduction}

In this paper, we will propose the visual matrix calculator named VISMAT, which has a basic matrix computation facility and an operations on a matrix such as elementary row and column operations. A user of VISMAT can make a matrix computation and matrix operation on a window as if he computes matrix calculations just using a notebook.

VISMAT is implemented in Java, so we may use it both as a frame based application and as an applet based application. Similar calculators are proposed such as NinfCalc using Ninf [1], TI-81 calculator [2], Point Five [3]. We can also find out such calculator through the Internet such as MatrixCalc. VISMAT differs from other calculators as follows. VISMAT displays a matrix element visually in a frame or an applet window. Then a user can calculate various computations on the matrix or the each element in the window. The matrix element may be a simple symbolic expression such as a fractional number. The purpose of VISMAT is mainly for education. We want to provide educational tool for an undergraduate student who will study an algorithm of basic matrix computations.

\section{Motivation and its brief history}

In order to educate undergraduate students in matrix computation algorithm and its programming, we have designed and implemented matrix computation language system named LAMAX[4][5][6][7]. LAMAX is an abbreviation for Language for Matrix. LAMAX is a programming language for matrix computation and it is implemented as a preprocessor to FORTRAN 77. In LAMAX, the user only writes a matrix expression directly in a program. Therefore, using LAMAX, students can make a program for solving various problems such as simultaneous linear equations, LU factorization, Eigenproblems, and so on in a shorter term. So they can understand the above methods clearly by making a 
program for themselves and can experience the characteristics of the methods using various type of data. In this sense, LAMAX is a very effective tool for education on computer programming of matrix computation. However the problem is such that students could not understand the elementwise operations of matrix computation because they only have to write a matrix expression and they don't have to mind elementwise operations at all. In some cases, there are very important situation where a student has to understand the elementwise operation as a basic concept such as gaussian elimination.

So we come to a conclusion that we need a new system which helps students for their understanding of the elementwise operation for matrix. In classical educational method, students are required practicing hard and hard by hand. For example, in the study of gaussian elimination, they are required solving a lot of problems using a notebook until they get used to do the operations. Although the operations are consisted of a lot of simple manipulations, the process is unreasonably tedious for the students. That is our motivation of VISMAT. It has a facility that can calculate matrix computation just as if the user do the same operations using a notebook. For example, VISMAT displays $7 \times 7$ matrix in the matrix display window, and enables us to do various elementwise operations. Figure 1 shows the illustration of VISMAT.

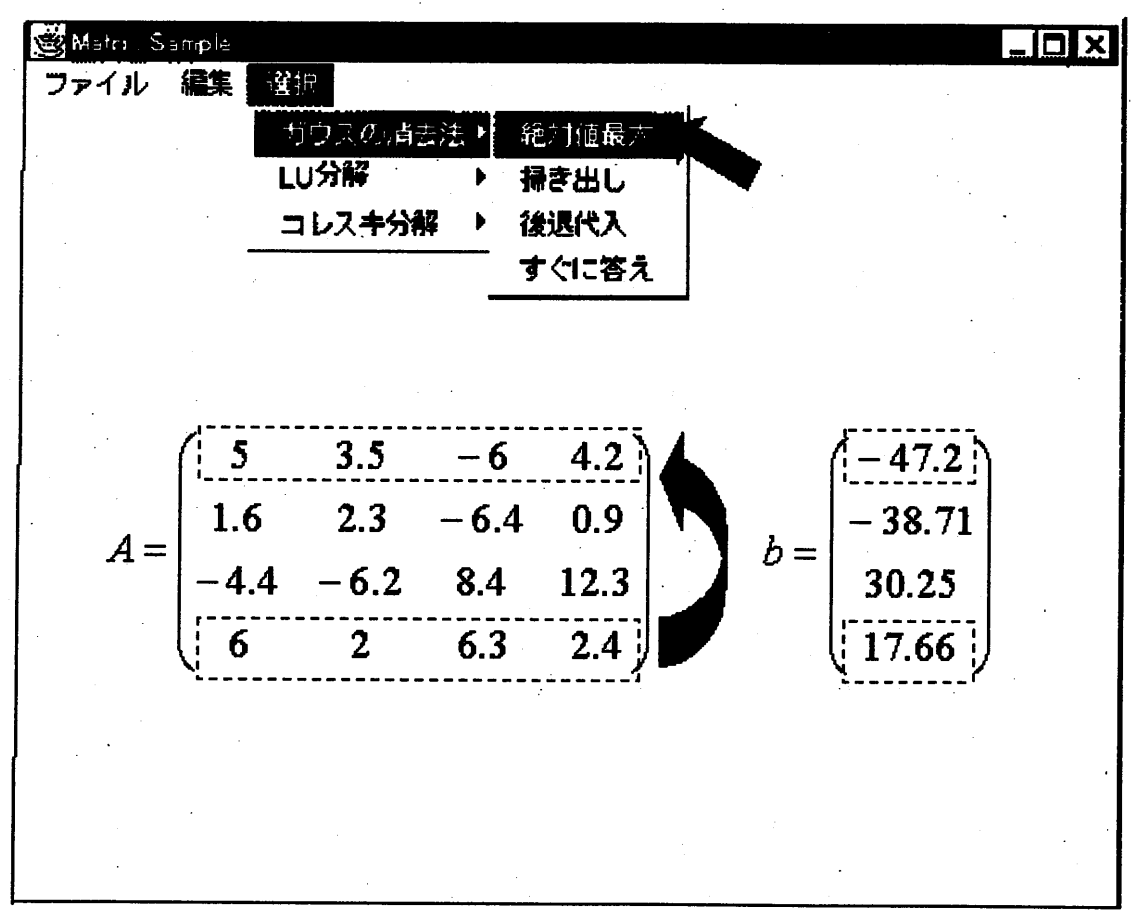

Figure 1: The VISMAT Window 


\section{The image of VISMAT using gaussian elimination}

In order to show the image of VISMAT more clearly, we introduce the outline of the VISMAT system using gaussian elimination algorithm. This is an algorithm for solving a simultaneous linear equations $A x=b$, where $A$ is the coefficient matrix, $b$ is the right hand side, and $x$ is the solution. Suppose that a student is required to solve the equation using a given data $A$ and $b$. First he inputs actual data of matrix $A$ and $b$ using an expression editor of VISMAT. In gaussian elimination algorithm, the following three steps are required: (1) Multiply a row through by a nonzero constant, (2) Interchange two rows, (3) Add a multiple of row to another row. These are called elementary row operations. As described before, these steps are comparative easy but unreasonably tedious. VISMAT provides the way that he can do the above steps just in a windows using graphical user interface.

The steps are explained in more detail. After inputting data, he has to find the absolute maximum value from the first column of the matrix $A$. To do this operation, he selects the first column of the matrix $A$ on the window using mouse, and select "find absolute maximum value" from the menu. Then the found value is highlighted. Then he interchanges the first row and a row which has the found value. Next, he may multiply the first row by $1 / a_{11}$ in order to introduce a leading 1 . Then, in order to eliminate, he adds suitable multiples of the top row to the rows below so that all entries below the leading 1 become zeros. All these steps are just done in the window visually and interactively. In this sense, we call the system as a visual matrix calculator.

As we limit the usage of VISMAT to education for undergraduate students, VISMAT accepts only at most $7 \times 7$ matrix. However as the acceptable size of matrix is limited, VISMAT may operate simple symbols and have a facility of symbolic execution.

\section{Conclusion}

We are now just implementing the system. After completing the system, we are going to use it in our introductory instruction on numerical analysis for undergraduate students. As there is a marked decline in the students' ability of mental calculation, we strongly believe that we will provide an environment where students can exercise in solving a various problem without unreasonable tedious labors.

VISMAT is implemented in Java as we described before, so it is executed as a frame window and an applet window through the Internet. This is very important for education. Recently, many educational tools are built as an applet, so we can easily combine such a tool with VISMAT.

VISMAT at present is only a calculator, but we have a plan to make it as a programming tool. VISMAT in near future will be a development environment for matrix computation.

As an application of this system, we may use VISMAT in the education of other field such as simplex tabular of Linear Programming. 


\section{References}

[1] S. Sekiguchi, M. Sato, H. Nakada, S. Matsuoka and U. Nagashima, - Ninf - : Network based Information Library for Globally High Performance Computing, Proc. of Parallel Object-Oriented Methods and Applications (POOMA), Santa Fe, Feb., 1996.

[2] Taylor, M. B., Watkins, W., Finding equations using calculator determinants, Mathematics and Computer Education, Vol. 27; No.3, (1993, fall) pp.276-279

[3] Marcoulides, G.A., Meecoulides, L.D., A review of Point Five, An Analysis And Modeling System, Vol. 13, No.3, (1987, Aug) pp.24-27

[4] S. Uchida, N. Yamaki, S. Hongo, Enhanced Programming Environment for Mathematical Software, Proceedings of the Third Conference of the Association of AsianPacific Operational Research Societies (APORS) within IFORS, pp. 469-476, World Scientific (1995.4)

[5] S. Uchida, N. Yamaki, S. Hongo, Y. Inoue, Y. Karasawa, The Architecture of A Programming Language LAMAX-S Oriented for the Mathematical Characteristics of Matrix, And Its Processor (In Japanese), Trans. of JSIAM, Vol. 2, No.3 (1992), pp. $155-168$

[6] S. Uchida, N. Yamaki, S. Hongo, Y: Karasawa, Y. Ikebe, The Optimum Code Generation For The Matrix Programming Language LAMAX-S (In Japanese), Trans. of JSIAM, Vol. 5, No.1 (1995), pp. 67-85

[7] S. Hongo, S. Uchida, LAMAX-E Users Guide (In Japanese), The Computer Centre, University of Tokyo (1984) 